\title{
Análisis del Funcionamiento del Algoritmo de Balanceo de Carga LCM en Redes de Conmutación de Etiquetas Multiprotocolo Generalizado (GMPLS)
}

\author{
Nancy Y. García*, Nelson E. Vera y Danilo A. López \\ Universidad Distrital "Francisco José de Caldas", Carrera 7 No. 40 - 53 Bogotá-Colombia. \\ (e-mail: nygelvezg@udistrital.edu.co, neverap@udistrital.edu.co, dalopezs@udistrital.edu.co) \\ * Autor a quien debe ser dirigida la correspondencia.
}

Recibido Jun. 25, 2014; Aceptado Ago. 19, 2014; Versión final recibida Sep. 10, 2014

\begin{abstract}
Resumen
El artículo presenta los resultados encontrados al evaluar el algoritmo de balanceo de carga LCM propio de MPLS para que sea usado como distribuidor de carga a través de distintos enlaces sobre redes de conmutación de etiquetas multiprotocolo generalizado (GMPLS), esto con el fin de evitar saturar la ruta primaria de transporte de datos entre el origen y destino. La metodología usada incluyó la implementación del algoritmo en $\mathrm{C}++$ para poder evaluar su desempeño en el simulador de eventos discretos (NS-2) y a partir de los resultados generar una propuesta de optimización que permitiera elevar su rendimiento. Se puede destacar a partir de las métricas evaluadas (rendimiento, fluctuación, nivel de balanceo y estabilidad) que LCM es un buen candidato para ser usado como distribuidor de carga en infraestructuras GMPLS.
\end{abstract}

Palabras clave: LCM, GMPLS, fluctuación, rendimiento, estabilidad, balanceo de carga.

\section{Performance Analysis of Load Balancing Algorithm LCM in Networks Generalized Multiprotocol Label Switching (GMPLS)}

\begin{abstract}
The article presents the results to evaluate the algorithm load balancing own LCM of MPLS to be used as load distributing through various links on switched networks generalized multiprotocol label (GMPLS), this in order to avoid saturating primary data transport route between the source and destination. The methodology used included the implementation of the algorithm in $\mathrm{C}++$ to evaluate their performance in discrete event simulator NS-2 and from the results generate an optimization proposal that would raise their performance. It can be noted from the metrics evaluated (throughput, jitter, level balancing and stability) that LCM is a good candidate as a distributor do load used in GMPLS infrastructure.
\end{abstract}

Keywords: LCM, GMPLS, jitter, throughput, stability, load balancing. 


\section{INTRODUCCIÓN}

Es claro que la tendencia del mundo globalizado se orienta hacia el concepto de que todo tipo de información sea enviada a través de la super-autopista de las comunicaciones llamada Internet; (López et al, 2014) esto ha comenzado a generar enormes cuellos de botella en los núcleos de la red debido a la descomunal cantidad de datos o flujos de información que deben procesar y reenviar los encaminadores y conmutadores; ello en parte gracias a la deficiente forma que tienen las tecnologías de networking de utilizar los recursos existentes. Todo ello ha generado que desde la industria y la misma academia se estén realizando investigaciones que permitan resolver muchas de las problemáticas que implican el envío y recepción del tráfico de manera óptima. En este sentido uno de los mayores avances a los que se ha llegado en los últimos años con el fin de resolver el problema del transporte óptimo de información se relaciona con el diseño (López et al, 2014) y desarrollo de estándares que eleven su rendimiento disminuyendo los recursos computacionales necesarios para su funcionamiento, entre los que se encuentran la conmutación de etiquetas multiprotocolo (MPLS) (López et al., 2012) y (GMPLS) (Weiqiang et al., 2012), éste último con la ventaja de transportar tanto datos a nivel eléctrico como óptico. No obstante de lo prometedor que pueda ser dicho estándar, existen varias líneas de investigación que aún se deben investigar entre las que se encuentra la aplicación de balanceo de carga de manera eficiente que tiene como finalidad la utilización de forma equilibrada de múltiples canales de transporte, al existir diferentes rutas desde un nodo origen de datos hasta uno destino y así poder hacer un uso más eficiente de los recursos sin saturarlos ni subutilizarlos evitando cuellos de botella.

LCM (Arco et al., 2004), es un algoritmo para reducir la congestión de la red evitando oscilaciones. Su objetivo es mantener el tráfico de la ruta principal en una banda estable comprendida entre el umbral de ocupación media $(M)$ y el umbral de congestión $(C)$ (Arco et al., 2004). Además de evitar oscilaciones en el reparto de tráfico, que se pueden dar cuando el camino de conmutación de etiquetas (LSP) principal y secundario, están congestionados (Arco et al., 2004) o cuando aparecen picos abruptos en los datos entregados por el usuario o la fuente generadora de datos. Desde el punto de vista real el valor de los umbrales son establecidos y configurados por el administrador de red, el umbral de congestión es configurado a un valor de carga máximo aceptable en los enlaces, que para el caso del artículo se estableció en $60 \%$ (Fig. 1) y su valor representa el límite a partir del cual el algoritmo empieza a transferir paquetes del LPS principal al secundario (Arco et al., 2004). El umbral (M) es el punto de retorno en el cual el algoritmo devuelve la carga del LSP secundario al primario (Arco et al., 2004). Bajo cualquier condición con LCM la carga de flujo del LSP primario siempre será mayor o igual que la del secundario.

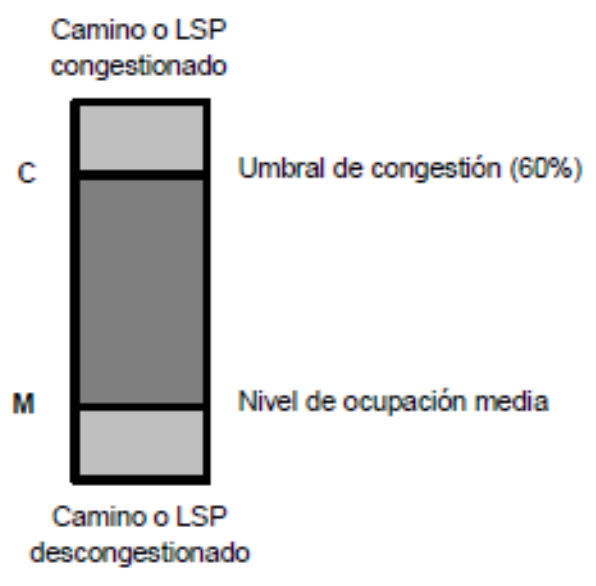

Fig. 1: Umbrales de uso en LCM.

El funcionamiento y diagrama de flujo del algoritmo es presentado en (Arco et al., 2004), de donde se destacan los siguientes aspectos:

LCM utiliza una variable llamada carga media $(L)$, pondera periódicamente tomando el flujo máximo que circula por las interfaces de salida de los conmutadores (LSRs) del LSP y el último valor de $L$, de acuerdo con la ecuación 1, donde, carga_de_salida_LSRij es el flujo recibido del LSRj del camino LSPi.

$C A(i)=\max \left(\right.$ carga_de_salida_LSR $\left.R_{i j}\right) \forall j \in 1 \ldots n$ 
Igualmente, LCM calcula periódicamente una variable llamada carga media $(L)$ tomando el flujo máximo de las interfaces de salida de los conmutadores (LSRs) que hacen parte de la red y el último valor de $L$, a partir de la ecuación 1; información que es enviada al encaminador de ingreso a la red (LER) que es quien obtiene el nivel de flujo actual $(C A)$ de cada LSP. La carga media $(L[t])$ es obtenida ponderando $C A$, con la constante $\alpha$, que es usada para controlar el peso de la carga actual frente a la historia del algoritmo, de acuerdo a la ecuación 2.

$L[t]=(1-\alpha) L[t-1]+\alpha C A$

donde, la constante $\alpha$ es muy importante cuando se evalúa LCM a nivel de estabilidad, valor que es determinado por el administrador de la red.

Este artículo propone la evaluación del algoritmo (Load balance with congestion and mean utilization thresholds) (LCM) utilizado en redes MPLS (conmutación de etiquetas multiprotocolo) para que sea probado en infraestructuras GMPLS (conmutación de etiquetas multiprotocolo generalizado) (El-Alfy et al., 2013), (Weiqiang et al., 2012), a fin de determinar su funcionamiento a partir de la evaluación de las variables de las red throughput (rendimiento), jitter (fluctuación), nivel de balanceo de carga y estabilidad de la red.

\section{MODIFICACION DEL ALGORITMO}

A partir de la migración del que se podría considerar como un estándar LCM (desarrollado inicialmente para su funcionamiento en redes MPLS) a estructuras GMPLS, las simulaciones presentaban ambigüedades en sus resultados desde la evaluación de las métricas: nivel de balanceo y jitter; en razón a que el algoritmo presentaba falencias cuando la carga media en el enlace secundario $(L s)$ era menor que la diferencia $M-$ $L p$ ( $L p$, carga media del enlace primario) y al requerirse mover la carga $M-L p$ del LSP secundario al primario, ya que el valor de Ls quedaba con un flujo negativo para la próxima iteración; situación ilógica que repercutía negativamente en el funcionamiento óptimo de LCM en redes GMPLS. La solución fue incluir el bloque de decisión (color oscuro) al diagrama de flujo original (Arco et al., 2004), quedando como se ve en la Fig. 2.

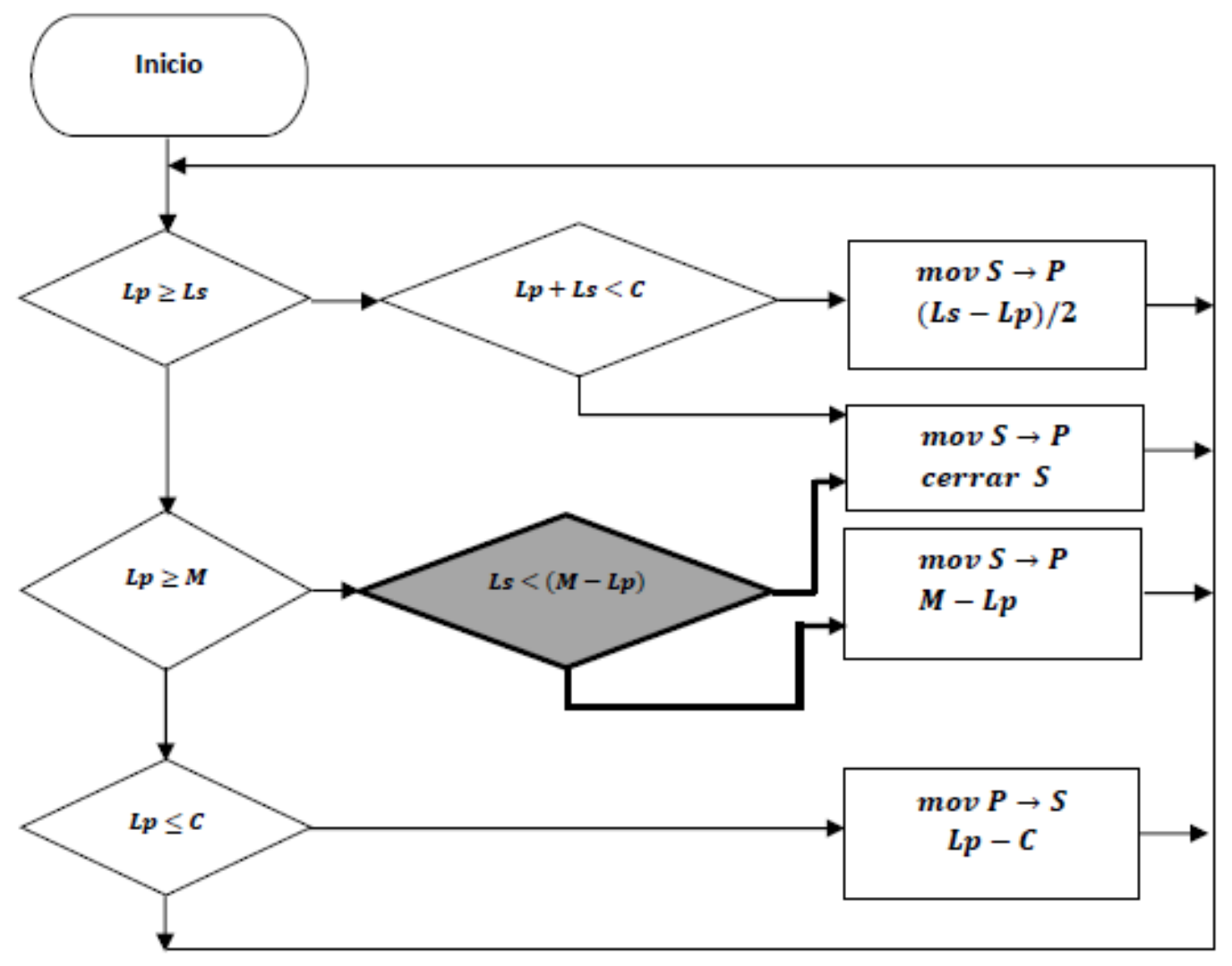

Fig. 2: Algoritmo LCM modificado de Arco et al. (2004) y Muñoz y Trujillo (2012) para su funcionamiento óptimo en GMPLS. 
Cuando $L p$ es mayor e igual a $L s$ y $L p$ mayor e igual que $M$ y menor e igual que $C$, es decir, cuando $L p$ este entre los umbrales $C$ y $M$, y $L p$ sea mayor que $L s$ el algoritmo no realiza ningún cambio en el tráfico. Si $L p$ está congestionado $(L p>C)$ pero $L s$ no lo está, se mueve la porción de tráfico $L p-C$ del LSP primario al LSP secundario. Si $L p$ es menor que $M$ (es decir el enlace esta subutilizado) y $L s$ es mayor que $M-L p$, entonces se mueve del LSP secundario al LSP primario la porción de tráfico $M-L p$. Si $L p$ está subutilizado (es decir es menor que $M$ ) y $L s$ es menor que $M-L p$, se debe mover todo el tráfico del LSP secundario al primario. Si $L p$ es menor que $L s$ y si la suma de $L p$ y $L s$ es menor que $C$ mover la totalidad del flujo del LSP secundario al primario. Si $L p$ es menor que $L s$ y la suma de $L p$ y $L s$ es mayor que $C$, mueva del LSP secundario al primario la porción de data $(L s-L p) / 2$.

\section{METODOLOGÍA Y ESCENARIO DE SIMULACIÓN}

La metodología utilizada para el análisis y evaluación del funcionamiento de LCM en ambientes GMPLS parte de una topología de red que es sobre el que se implementa y simula el algoritmo, utilizando los lenguajes de programación C++ y TCL propios del simulador de eventos discretos Network Simulator (NS2), para seguidamente estudiar su funcionamiento desde el punto de vista de las métricas de rendimiento, jitter, nivel de balanceo y estabilidad a partir de la transferencia de datos (López et al., 2012).

La topología GMPLS de red que se usó para el desarrollo de la investigación (ver Fig. 3) está formada por 8 nodos, donde los conmutadores ópticos son los que aparecen identificados como OXC (cabe destacar que fue necesario implementarlos en código al igual que la nube GMPLS porque el simulador no los soportaba), los LER corresponden a encaminadores electro-ópticos y los servidores son los que generan y reciben la carga. El flujo de datos que circula por la red es de tipo eléctrico y óptico, donde la tasa de transferencia desde el emisor al receptor es de 8 Mbps y la capacidad de cada ruta es las mostradas en la Fig. 3. Con el fin de evaluar el algoritmo de balanceo LCM se tomó como punto de partida la posibilidad de distribuir la carga a través de dos caminos distintos (que corresponde al máximo de enlaces para aplicar) de conmutación de etiquetas bidireccionales conocidos como FA-LSPs (que son los enlaces virtuales que se deben establecer para el transporte de los datos por cada canal físico) desde el LER-1. Estos fueron identificados así: i) Camino 1 (LSP1): LER-1 _ OXC-1 _ OXC-2 _ LER-2; y ii) Camino 2 (LSP2): LER-1 _ OXC-3_OXC-4_LER-2

El retardo de cada medio de transporte es variable y depende de los valores que pueda tomar la variable $b i$ (que esta entre 0 y $10 \mathrm{mseg}$ ) y cambia de acuerdo al parámetro que se esté evaluando.

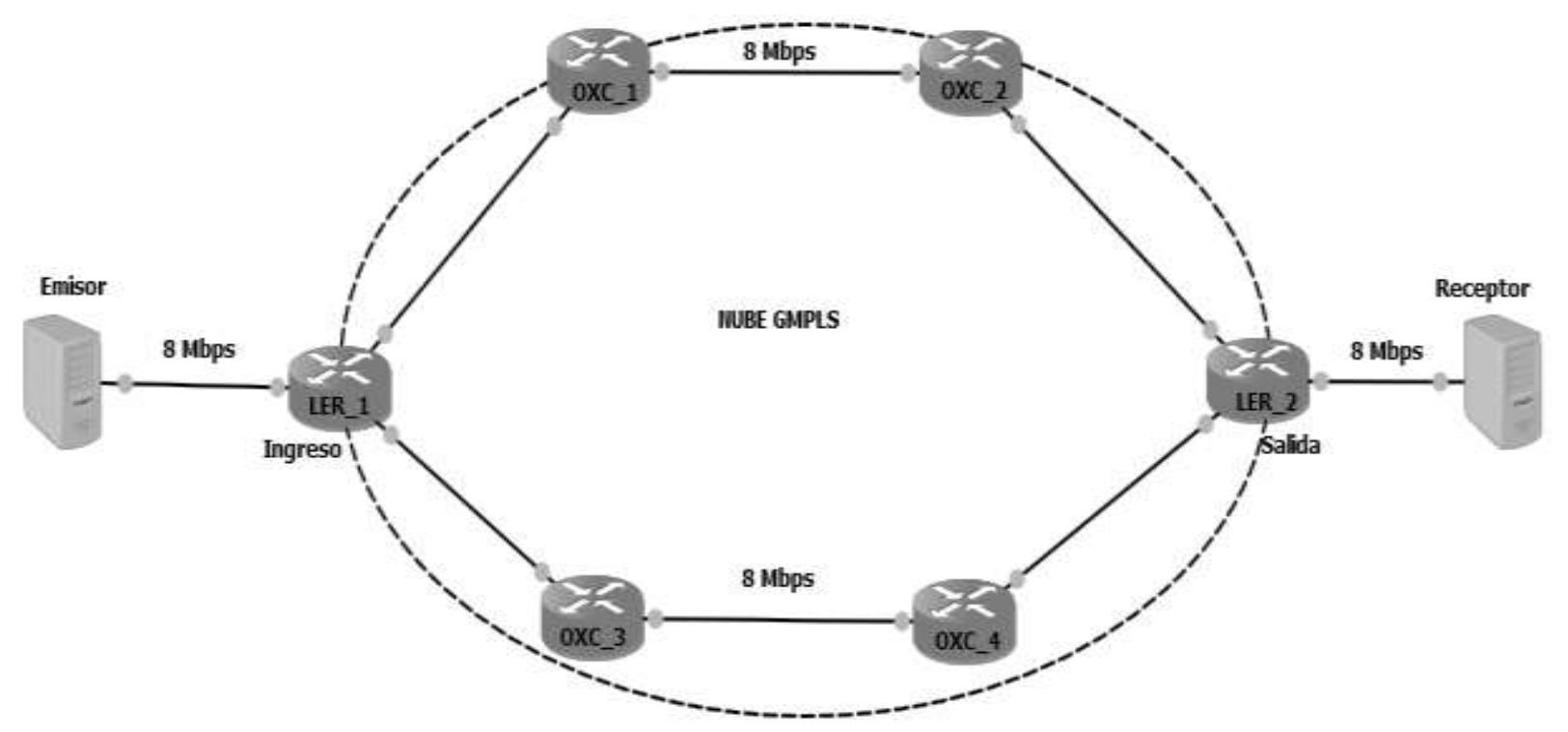

Fig. 3: Estructura de la red GMPLS.

\section{RESULTADOS Y ANALISIS}

La estimación del funcionamiento de LCM en GMPLS se llevó a cabo a través de la evaluación del desempeño de las métricas: rendimiento, fluctuación (jitter), nivel de balanceo y estabilidad, que son los parámetros que dan una idea de que tan robusto es un distribuidor de carga en las redes teleinformáticas (Anti et al., 2012). 


\section{Utilización de la red}

Medida que indica en un instante de tiempo el volumen de tráfico que está siendo transmitido por un LSP (Zhanqui et al., 2013). Durante el análisis de esta métrica se establece que los enlaces de los LSPs no están congestionados es decir es inferior al $60 \%$ de la capacidad del enlace (umbral de 0,6); el reparto de carga se realiza en una cantidad máxima de tráfico por el LSP primario igual al umbral de congestión de la capacidad del LSP, si el tráfico entrante es mayor que el umbral de congestión, el excedente se envía por el LSP secundario, pero si es menor que el nivel de congestión todo el caudal se transmite por el LSP primario sin aprovechar el camino inutilizado (característica propia de funcionamiento de LCM).

En la Fig. 4, se muestra el comportamiento de cada LSP frente al caudal enviado (TRAF1 y TRAF2) y calculado por el algoritmo LCM. El tráfico por el LSP1 se fija en un valor máximo para evitar congestión por debajo de 6 Mbps. Cuando el emisor comienza a generar datos se observa que la capacidad del LSP1 va aumentando de forma paralela a la secuencia de información que se envía por allí, comportamiento que se observa hasta los $7 \mathrm{seg}$, no obstante el enlace LSP2 (que es el que entraría a funcionar en caso de saturación) permanece inactivo durante este periodo de tiempo. Alcanzado el umbral (alrededor de los 7 seg) LCM detecta la existencia de saturación por ese enlace, lo que obliga a enviar parte de la data por el LSP2 incrementando su nivel a medida que transcurre el tiempo y dependiente de la cantidad de información que se esté entregando desde el usuario emisor. A partir de los 26 seg la secuencia de datos comienza a disminuir hasta un t=34 seg cayendo el caudal del LSP2 a cero, en razón a que LCM aplica balanceo solo si el LSP primario esta congestionado, es decir, si supera el umbral, en caso contrario solo envía paquetes por el LSP primario sin utilizar los demás recursos disponibles de la red, propiedad favorable si se tiene en cuenta que podría ser utilizado por otros clientes de la red.

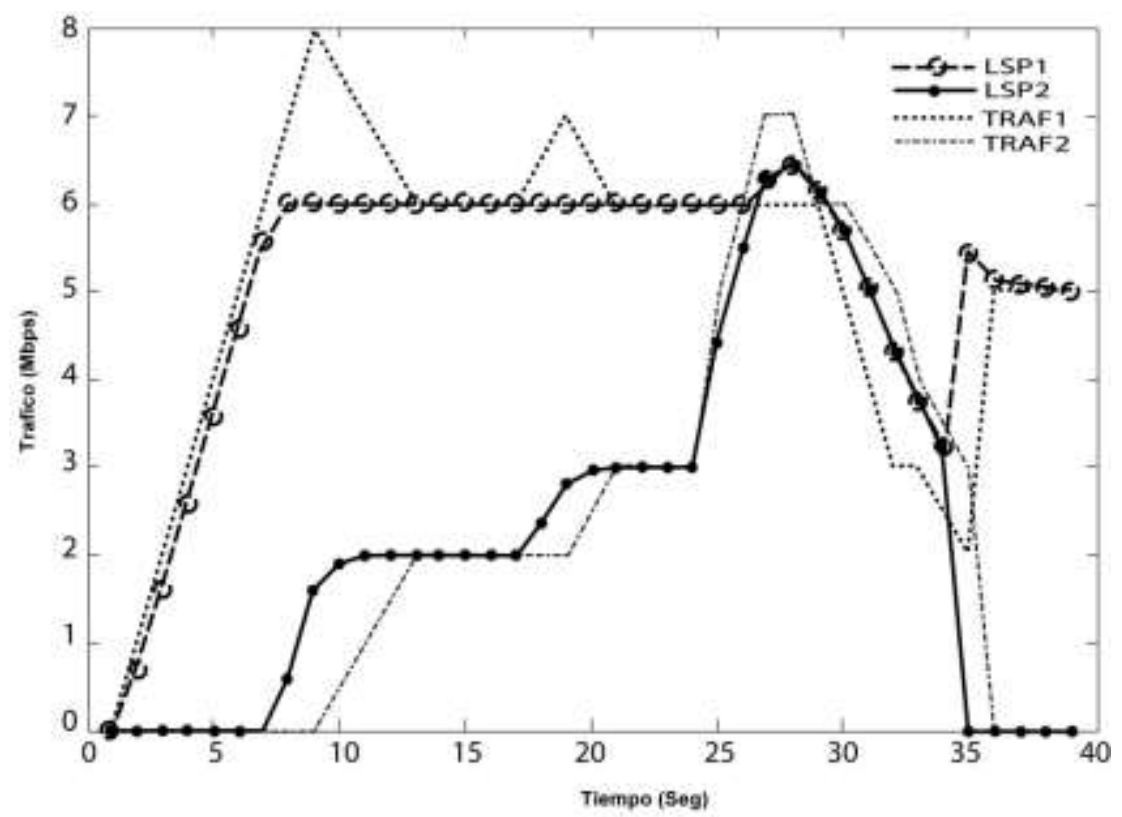

Fig. 4: Tráfico medido y tráfico asignado a cada LSP.

\section{Jitter}

Variable que se refiere a la fluctuación de los retardos paquete a paquete (Demichels y Chimento, 2002). Para su cálculo se toman los valores absolutos de cada uno de los valores del Jitter de paquete a paquete, porque al existir valores negativos se restarían, afectando su cálculo promedio, es decir lo importante es la variabilidad en valor absoluto de jitter. La ecuación matemática que se implementó en la topología de la red para estudiar esta métrica de evaluación es la mostrada en la ecuación 3 (Chih-Heng, K, 2011).

$$
\text { Jitter }=\text { Factor Jitter }\left(t_{\text {proc }}+t_{\text {trans }}\right)
$$

donde, Factor Jitter es un número proporcional del tiempo de procesamiento $\left(t_{\text {proc }}\right)$ más el tiempo de trasmisión $\left(t_{\text {trans }}\right)$ que le toma al router frontera (identificado en la Fig. 2 como LER_1) procesar y transmitir un paquete. Este factor multiplicado por este tiempo da el valor del Jitter. 
En la Fig. 5, se observa el comportamiento cualitativo de la variación de los LSP1 y LSP2 respectivamente producido por el reparto de tráfico mediante flujos (a partir de la dirección IP o puerto) con el algoritmo de balanceo de carga LCM. Su valor va a depender de cuan mezclados estén los flujos, pues a mayor aleatoriedad será más alto; si por el contrario la información se mantiene con un número de paquetes seguidos de una misma fuente de información este nivel promedio será menor. Para simular el proceso se genera tráfico de forma aleatoria usando diferentes aplicaciones (video, audio, datos) y se clasifica dependiendo de si el límite Hash CRC (Cao et al., 2001) es mayor o menor. Los resultados cuantitativos aparecen en la tabla 1.

Se observa que el jitter en el LSP2 fue mayor que en el LSP primario y se presentó un retardo promedio paquete a paquete de 5.78 mayor que el retardo promedio del otro camino debido a que la tasa de tráfico enviada fue pequeña de $16.6 \%$. Si la distribución del total de los paquetes entre la cantidad de flujos es uniforme (es decir cada flujo tiene el mismo número de paquetes) en el LER_1, el límite de Hash se incrementará provocando un nivel de balanceo del $50 \%$ por cada enlace o medio de transporte como se muestra en la Fig. 6 y tabla 2.
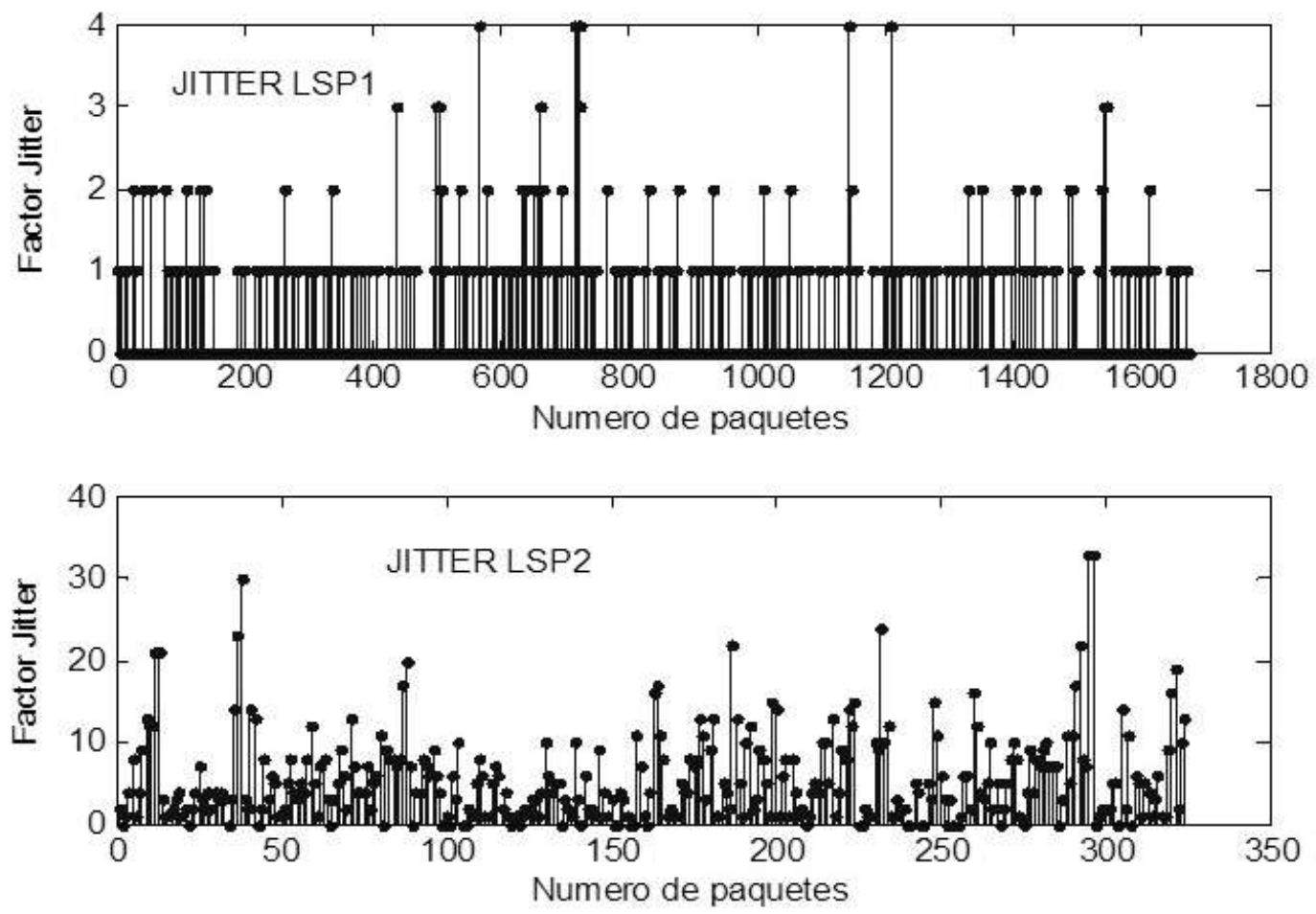

Fig. 5: Jitter por los LSP, cuando se genera tráfico aleatorio.

Tabla 1: Valores cuantitativos para tráfico aleatorio.

\begin{tabular}{|l|c|c|}
\hline \multicolumn{1}{|c|}{ Variable } & LSP1 & LSP2 \\
\hline Número de paquetes (2000) & 1675 & 325 \\
\hline Número de flujos & 12 & 12 \\
\hline $\begin{array}{l}\text { Limite hash (taza de tráfico a repartir por el camino de } \\
\text { respaldo LSP_2) }\end{array}$ & $-\cdots---$ & $16.6 \%$ \\
\hline Factor Jitter & 0.3360 & 5.3900 \\
\hline Retardo promedio & $1.2071 \mathrm{~ms}$ & $5.7881 \mathrm{~ms}$ \\
\hline
\end{tabular}

Tabla 2: Valores cuantitativos para tráfico uniforme.

\begin{tabular}{|l|c|c|}
\hline \multicolumn{1}{|c|}{ Variable } & LSP1 & LSP2 \\
\hline Número de paquetes (2000) & 1000 & 1000 \\
\hline Número de flujos & 12 & 12 \\
\hline $\begin{array}{l}\text { Limite hash (taza de tráfico a repartir por el camino de } \\
\text { respaldo LSP2) }\end{array}$ & ---- & $50 \%$ \\
\hline Factor Jitter & 1.2903 & 1.3384 \\
\hline Retardo promedio & $1.9772 \mathrm{~ms}$ & $2.0172 \mathrm{~ms}$ \\
\hline
\end{tabular}



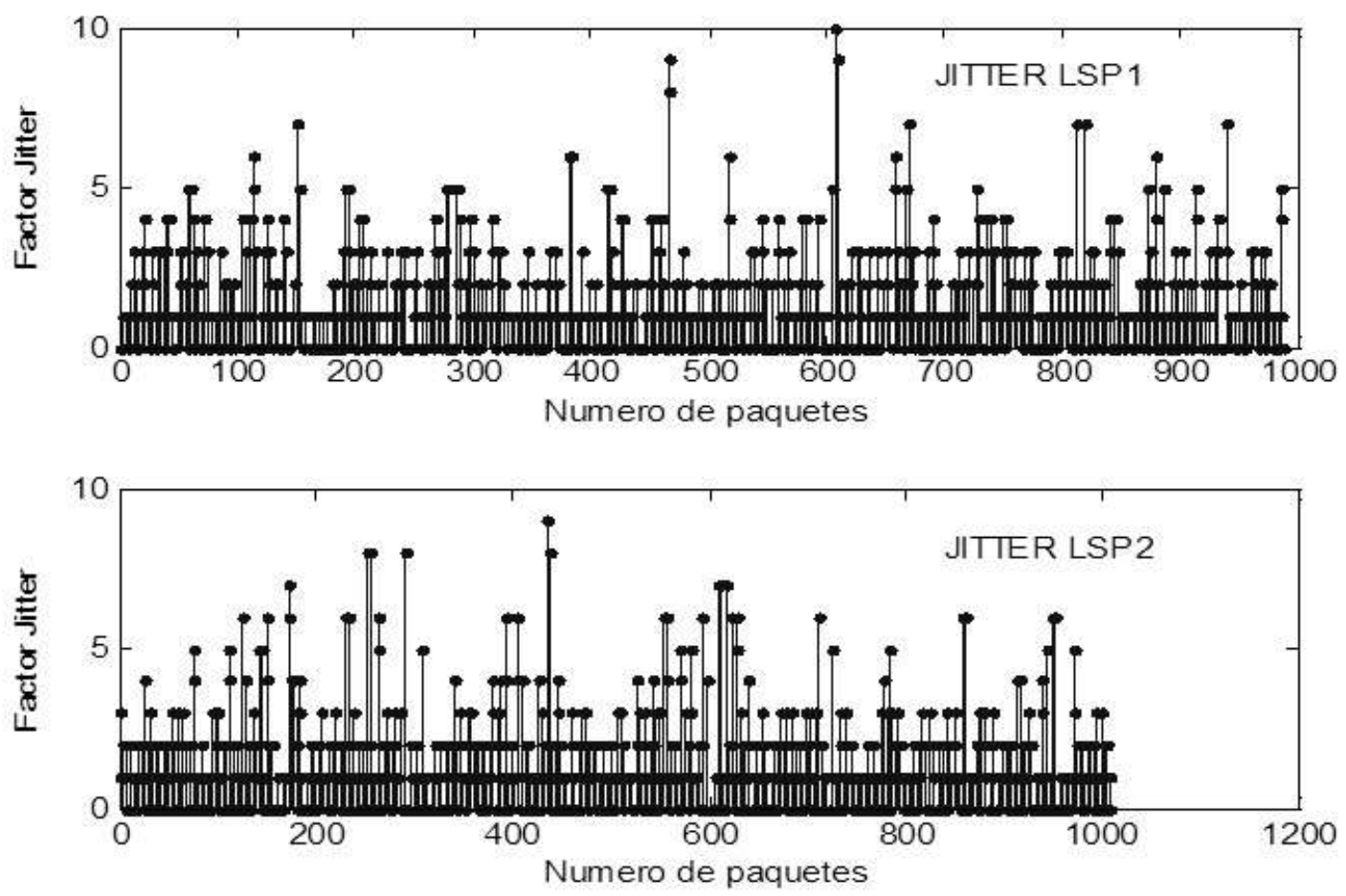

Fig. 6: Jitter por los LSP, cuando se genera tráfico uniforme.

En la tabla 3, se relacionan los porcentajes de fluctuación y retardo promedio por cada camino de conmutación de etiquetas en GMPLS para diferentes valores Hash. Se puede concluir que a mayor porcentaje de reparto de carga, menor retardo promedio y mayor valor del Jitter por el LSP2.

En la tabla 4 se compara el comportamiento que presenta el balanceo de carga LCM frente al ofertado por el algoritmo MATE (López et al., 2014). Se puede observar que entre menos porcentaje de reparto de carga, el retardo promedio aumenta en los dos algoritmos; la fluctuación en el algoritmo MATE a través del LSP_2 es menor comparado con el jitter que presenta el algoritmo LCM, en razón al modo en el que realiza el algoritmo el reparto de carga; en el primer caso basado en paquetes y el segundo basado en la conexión o por flujos.

Tabla 3: Jitter y retardo promedio por los LSPs con LCM.

\begin{tabular}{|l|l|c|c|c|c|}
\hline \multirow{2}{*}{$\%$ LSP1 } & \multirow{2}{*}{$\%$ LSP2 } & \multicolumn{2}{|c|}{ Jitter (ms) } & \multicolumn{2}{c|}{ Retardo promedio (ms) } \\
\cline { 3 - 6 } & & LSP1 & LSP2 & LSP1 & LSP2 \\
\hline $90 \%$ & $10 \%$ & 10.324 & 0.1827 & 10.911 & 1.0996 \\
\hline $80 \%$ & $20 \%$ & 4.435 & 0.4552 & 4.71 & 1.27 \\
\hline $70 \%$ & $30 \%$ & 2.6184 & 0.688 & 3.2775 & 1.4384 \\
\hline $60 \%$ & $40 \%$ & 1.8162 & 0.9678 & 2.4443 & 1.6884 \\
\hline $50 \%$ & $50 \%$ & 1.3466 & 1.376 & 1.988 & 2.011 \\
\hline
\end{tabular}

Tabla 4: Jitter y retardo promedio por los LSPs con los algoritmos LCM y MATE.

\begin{tabular}{|c|c|c|c|c|c|c|c|c|c|}
\hline \multirow{3}{*}{ \% LSP1 } & \multirow{3}{*}{ \%LSP2 } & \multicolumn{4}{|c|}{ MATE } & \multicolumn{4}{|c|}{ LCM } \\
\hline & & \multicolumn{2}{|c|}{ Jitter (ms) } & \multicolumn{2}{|c|}{$\begin{array}{l}\text { Fluctuación } \\
\text { promedio (ms) }\end{array}$} & \multicolumn{2}{|c|}{ Jitter (ms) } & \multicolumn{2}{|c|}{$\begin{array}{l}\text { Fluctuación } \\
\text { promedio (ms) }\end{array}$} \\
\hline & & LSP1 & LSP2 & LSP1 & LSP2 & LSP1 & LSP2 & LSP1 & LSP2 \\
\hline $90 \%$ & $10 \%$ & 0.2219 & 0.1827 & 0.2219 & 0.2219 & 10.324 & 0.2219 & 10.911 & 1.0996 \\
\hline $80 \%$ & $20 \%$ & 0.5 & 0.4552 & 0.5 & 0.5 & 4.435 & 0.5 & 4.71 & 1.27 \\
\hline $70 \%$ & $30 \%$ & 0.8577 & 0.688 & 0.8577 & 0.8577 & 2.6184 & 0.8577 & 3.2775 & 1.4384 \\
\hline $60 \%$ & $40 \%$ & 0.9678 & 0.6661 & 0.6661 & 0.6661 & 1.8162 & 0.6661 & 2.4443 & 1.6884 \\
\hline $50 \%$ & $50 \%$ & 1.376 & 0 & 0 & 0 & 1.3466 & 0 & 1.988 & 2.011 \\
\hline
\end{tabular}




\section{Nivel de balanceo}

Permite conocer la proporción de datos respecto del total enviado que se transmite por un camino, y se calcula como la cantidad de información que se va a repartir por los caminos bidireccionales LSPs de la red GMPLS dividido por el volumen total de entrada al LER en el núcleo de la networking (Wenda et al., 2013). Como es una medida en proporción del tráfico total, se da en términos de porcentaje y su ecuación se relaciona en 4 (López et al., 2014).

$$
N B \%=\frac{\text { tráfico a repartir }}{\text { tráfico total }} * 100
$$

Para el análisis de esta métrica, se evalúa simultáneamente los resultados obtenidos al graficar el nivel de tráfico y balanceo de carga por los dos enlaces (LSP1 y LSP2). En el primer caso (Fig. 7) el LSP1 es quien tiene la prioridad de transferir datos mientras no se llegue al umbral crítico de congestión del $60 \%$, caso que se evidencia durante el intervalo de los 0 a $7 \mathrm{seg}$, pues el $100 \%$ de la información que se está transmitiendo circula por ese enlace; por el LSP2 el caudal es de $0 \%$, comportamiento que es corroborado en la Fig. 8.

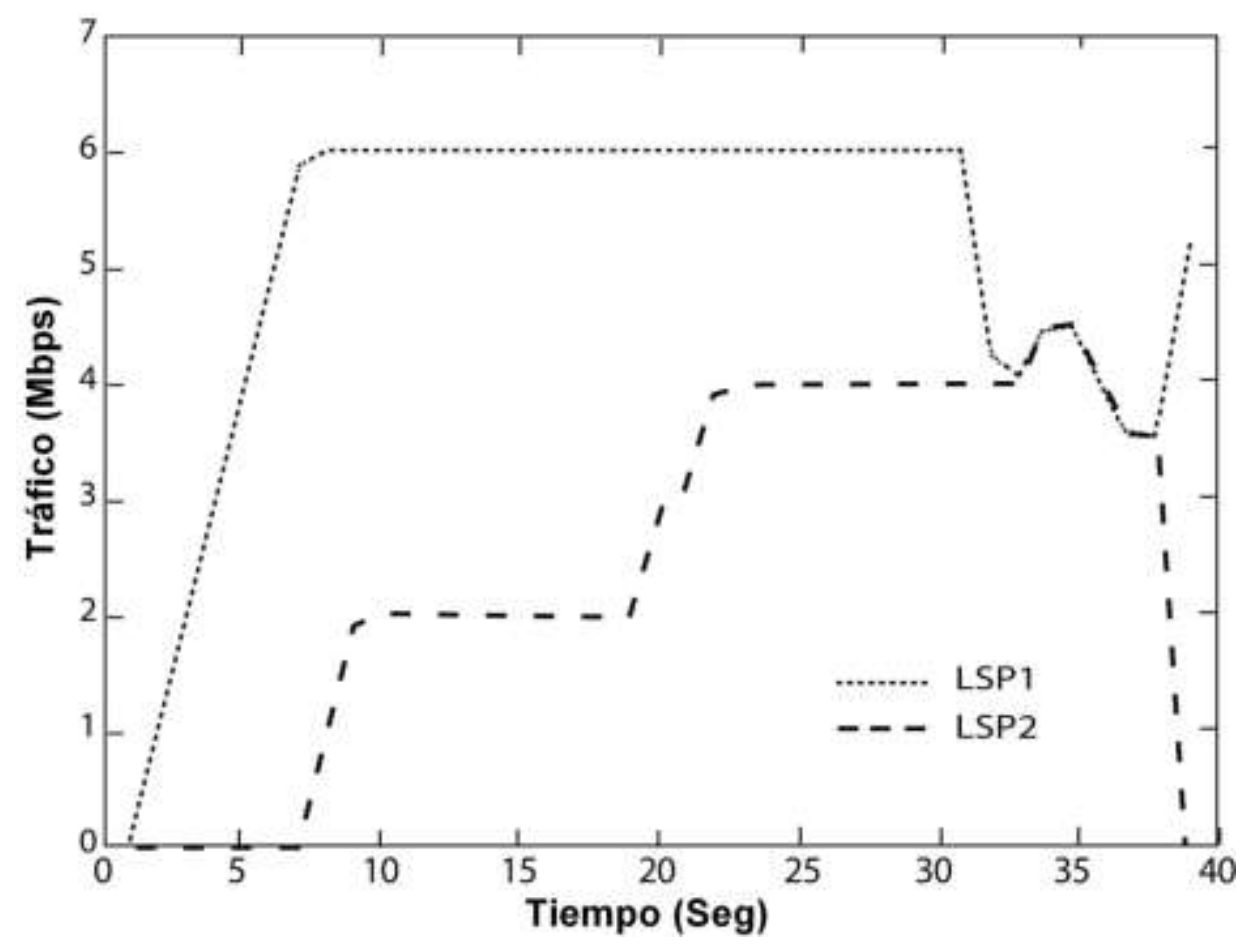

Fig. 7: Tráfico por cada LSP cuando el flujo es desigual en ambas rutas.

Durante el periodo de los 7 a $18 \mathrm{seg}$, el nivel de balanceo comienza a variar de entre $0 \%$ y $100 \%$ a $75 \%$ y el $25 \%$ respectivamente, que es cuando comienza a descongestionarse el LSP primario y el algoritmo envía ese exceso de datos por el LSP2; en el intervalo de los 22 a $31 \mathrm{seg}$, el balanceo es más óptimo alcanzando un $40 \%$ por el LSP2, hasta llegar a un $50 \%$ lo que garantiza una utilización uniforme de los recursos de la red sin generar congestión en ninguno de los dos enlaces. Nótese que en cualquier instante de tiempo el nivel de carga es del $100 \%$ lo que indica que siempre y cuando exista disponibilidad de ancho de banda no habrá descarte de paquetes en las colas de procesamiento. Adicionalmente se observa que existe una concordancia en cuanto al funcionamiento de LCM en redes GMPLS y que aunque su comportamiento no es explicito (debido a que en LCM los datos a transportar no se asignan de forma previa a un camino virtual, sino que se van transfiriendo porciones de un LSP a otro dependiendo del umbral de saturación) funciona adecuadamente con este tipo de tecnologías que se podrían considerar ópticas.

Para el segundo caso, entre los 23 y 39 seg (Fig. 9), el flujo es igual en ambas rutas, con un balanceo del $50 \%$ en cada LSP (Fig. 10); adicionalmente en la Fig. 9, el umbral de congestión está en el $60 \%$, por ello el tráfico por el LSP1 es de 6 Mbps, mientras que por el LSP2 está por debajo del valor de congestión; cuando la cantidad de datos total entrante supera los $12 \mathrm{Mbps}$ se reparten equitativamente a partir de $\mathrm{t}=24$ seg; también se puede apreciar que en el intervalo $t=24$ a $29 \mathrm{seg}$, los LSPs están superando el umbral de 
congestión (son mayores a 6 Mbps que es el umbral de $60 \%$ de la capacidad del enlace de 12 Mbps) produciéndose perdida de información. El comportamiento del tráfico entrante es creciente hasta $\mathrm{t}=26 \mathrm{seg}$, donde llega hasta los $14 \mathrm{Mbps}$ y desde donde empieza a mostrarse un comportamiento decreciente producto de la disminución del envío de datos desde el emisor.

La gráfica de balance de carga para la distribución de tráfico de la Fig. 9, se encuentra en la Fig. 10, donde se destaca que a medida que se va asignando más tráfico al LSP2 y mientras el LSP1 se encuentra en el umbral de congestión, el nivel de balanceo crece para el LSP secundario y decrece para el primario que se acerca en función del tiempo al $50 \%$ del flujo entrante para cada LSP.

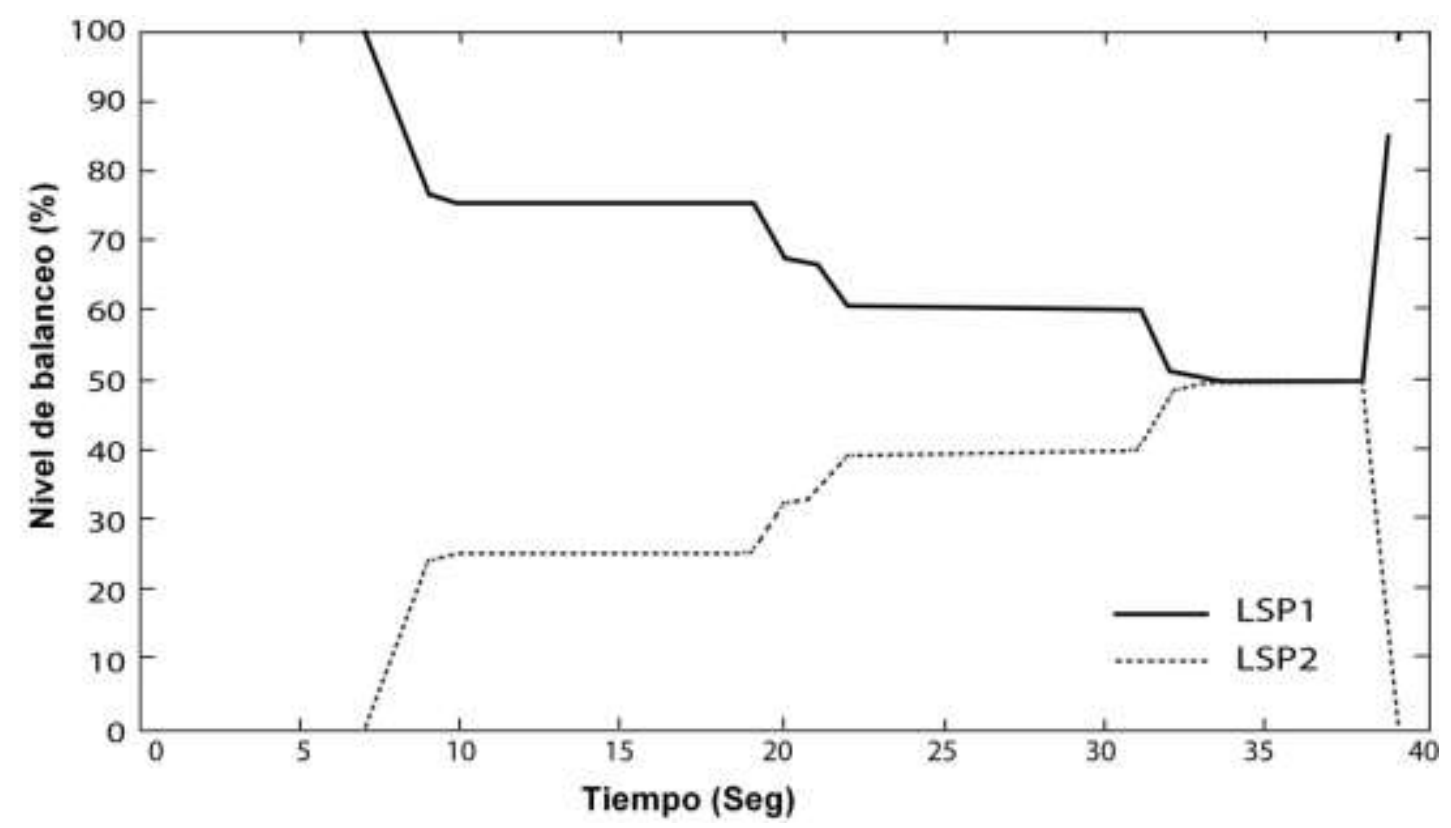

Fig. 8: Nivel de balanceo con LCM cuando el flujo de datos es desigual por ambas rutas.

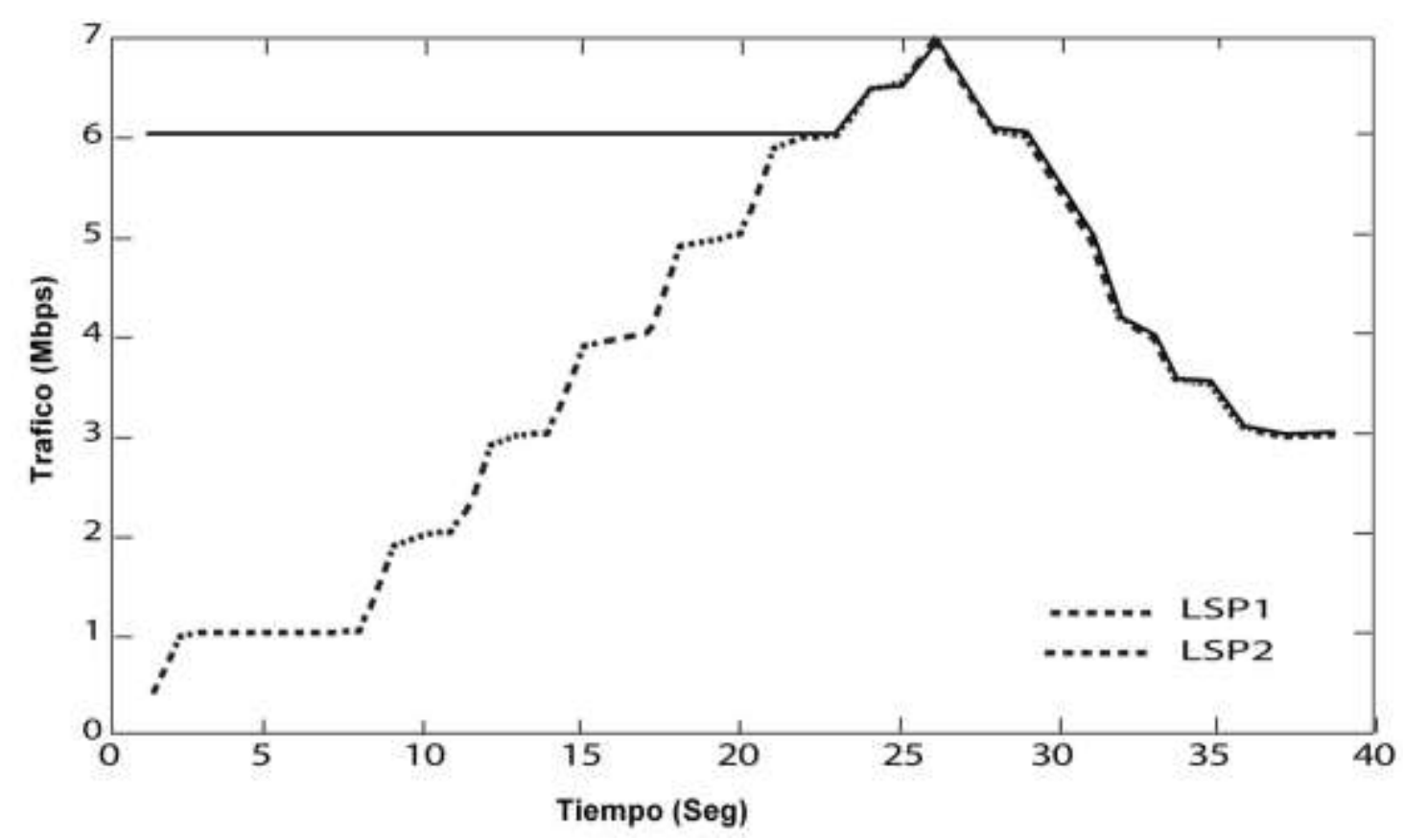

Fig. 9: Tráfico por cada LSP para LCM cuando el flujo es igual en ambas rutas. 


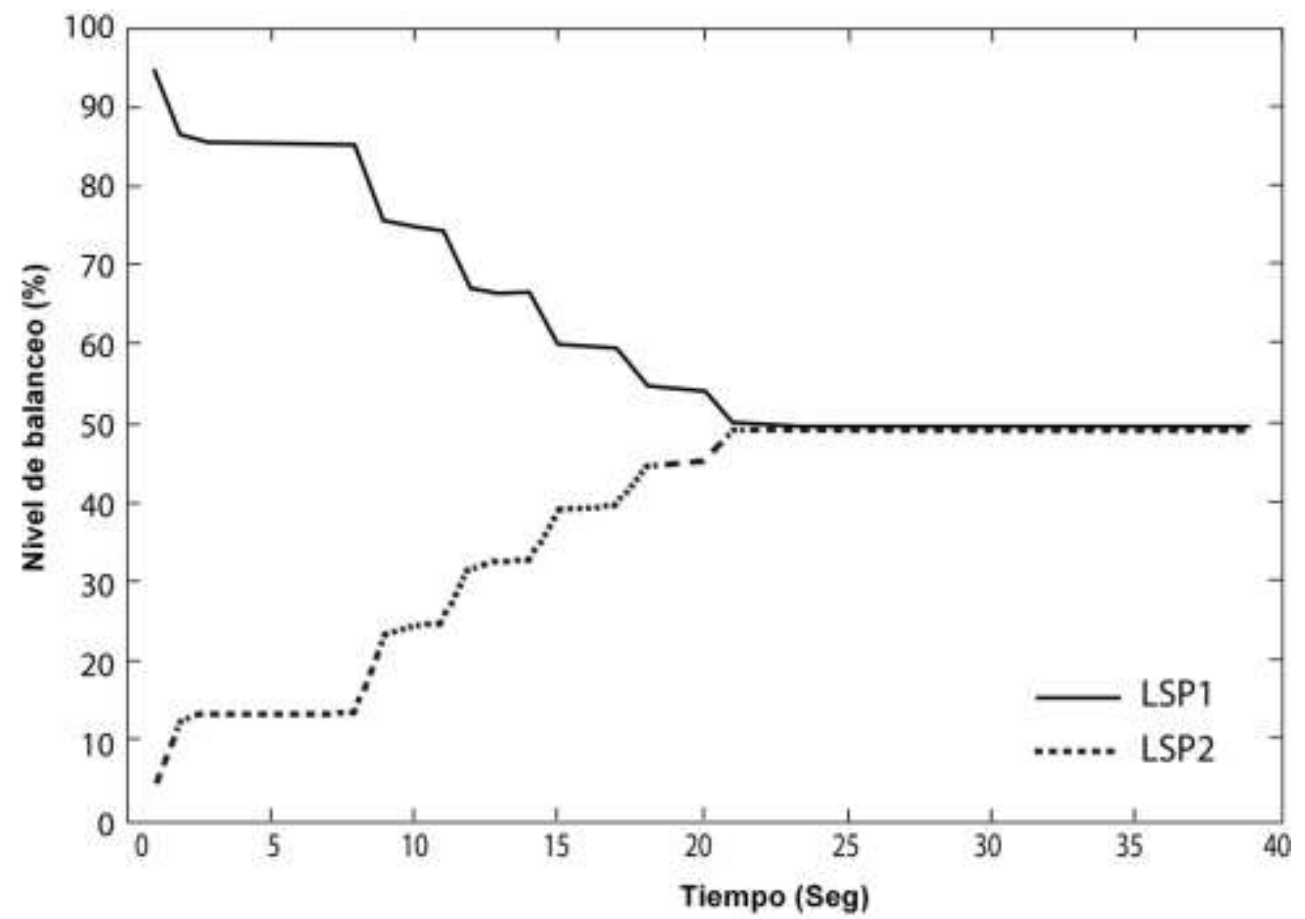

Fig. 10: Nivel de balanceo para LCM cuando el flujo de datos es igual por ambas rutas.

Como elemento de contraste y evaluación en la tabla 5 se muestra el comportamiento cuando LCM y MATE realizan el reparto de carga (en porcentajes) a medida que transcurre el tiempo, para los LSPs de la Fig. 3.

Tabla 5: Porcentaje de balanceo de carga en LCM y MATE sobre redes GMPLS.

\begin{tabular}{|l|c|c|c|c|}
\hline \multirow{2}{*}{$\begin{array}{c}\text { Tiempo } \\
\text { (seg) }\end{array}$} & \multicolumn{2}{|c|}{ LCM } & \multicolumn{2}{c|}{ MATE } \\
\cline { 2 - 5 } & LSP1 & LSP2 & LSP1 & LSP2 \\
\hline 5 & $100 \%$ & $0 \%$ & $100 \%$ & $0 \%$ \\
\hline 10 & $75 \%$ & $25 \%$ & $70 \%$ & $30 \%$ \\
\hline 20 & $61 \%$ & $39 \%$ & $40 \%$ & $60 \%$ \\
\hline 30 & $41 \%$ & $59 \%$ & $50 \%$ & $50 \%$ \\
\hline 40 & $50 \%$ & $50 \%$ & $50 \%$ & $50 \%$ \\
\hline
\end{tabular}

A partir del análisis se puede concluir que le toma alrededor de 30 seg estabilizar la respuesta a MATE, tiempo en el cual los flujos que disminuyen automáticamente se están asignando al otro LSP compensando así de forma eficiente la carga sin pérdida de información, factor que también es cumplido por LCM pero con mayores retardos debido a la mayor oscilación existente. Lo anterior sugiere que MATE realiza un reparto de tráfico muy eficiente a través de las rutas disponibles donde en cualquier instante de tiempo, siempre la suma de los porcentajes de equilibrio serán iguales al $100 \%$ del total que está ingresando al LER-1 (López et al, 2014).

\section{Estabilidad}

Métrica crítica dentro de un mecanismo de balanceo de carga en tiempo real ya que es susceptible a cambios impredecibles en la red como caída en los enlaces, reconfiguración de las tablas de routing, rutas más óptimas a destinos; dichas oscilaciones a veces bruscas hacen que la respuesta de un balanceador responda de forma inestable generando transiciones que pueden ser rápidas o lentas (ver Fig. 11) en el tiempo, en algunos casos en proporción a las transiciones ocurridas en la entrada produciendo un rendimiento bajo en la capacidad de reparto ya que pueden ocasionar aumentos en el jitter. No obstante algunos balanceadores como el presentado en el artículo poseen parámetros en sus modelos funcionales como constantes elegibles (llamados factores de convergencia $\alpha$ ) que pueden llegar a controlar estas variaciones en la respuesta cuando se presenten. Para evaluar la estabilidad de LCM se generan oscilaciones en el valor de las tasas de tráfico, cuando $\alpha$ toma valores de $1,0.5$ y 0.1 analizando en cada caso cómo ésta conducta afecta a los LSPs y al nivel de balanceo, lo que provoca que el sistema global converja a un punto óptimo (como el mostrado en la Fig. 16). 
Los resultados arrojados una vez simulada la red (Fig. 11) muestran la fluctuación en la data (TRAF1, TRAF2) y la forma en que se reparte la carga calculada a transportarse por los caminos virtuales LSP1 y LSP2. Se observa que la respuesta (actualizaciones) de LCM para un $\alpha=1$ en los LSPs es muy rápida tratando de adaptarse a las mismas variaciones pico del flujo de datos, generando una inestabilidad importante en el sistema, conducta indeseable en la red.

La Fig. 12 describe las variaciones generadas en el nivel de balanceo para los LSPs como función del tiempo. A partir de su comportamiento se concluye que con $\alpha=1$ el algoritmo no toma en cuenta las mediciones realizadas con anterioridad, únicamente se vale de las actuales para realizar y aplicar los cálculos; por ello la respuesta es tan rápidamente oscilante en los LSPs generando inestabilidad; específicamente esta situación se presenta en el periodo que va de los 5 a los $23 \mathrm{seg}$, donde se aparecen una serie de vibraciones en la gráfica de balanceo de carga, respuesta poco atractiva que degrada el rendimiento en la distribución de los paquetes.

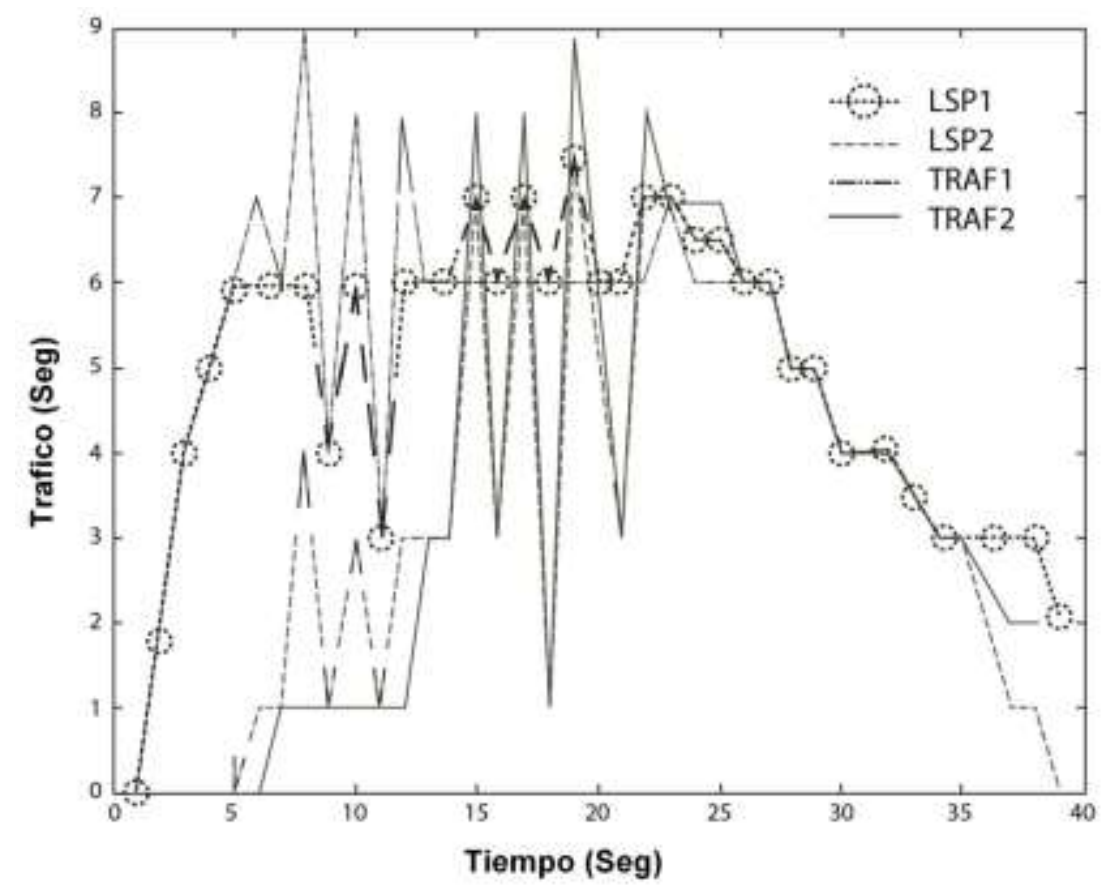

Fig. 11: Inestabilidad en el tráfico por los LSP $(\alpha=1)$.

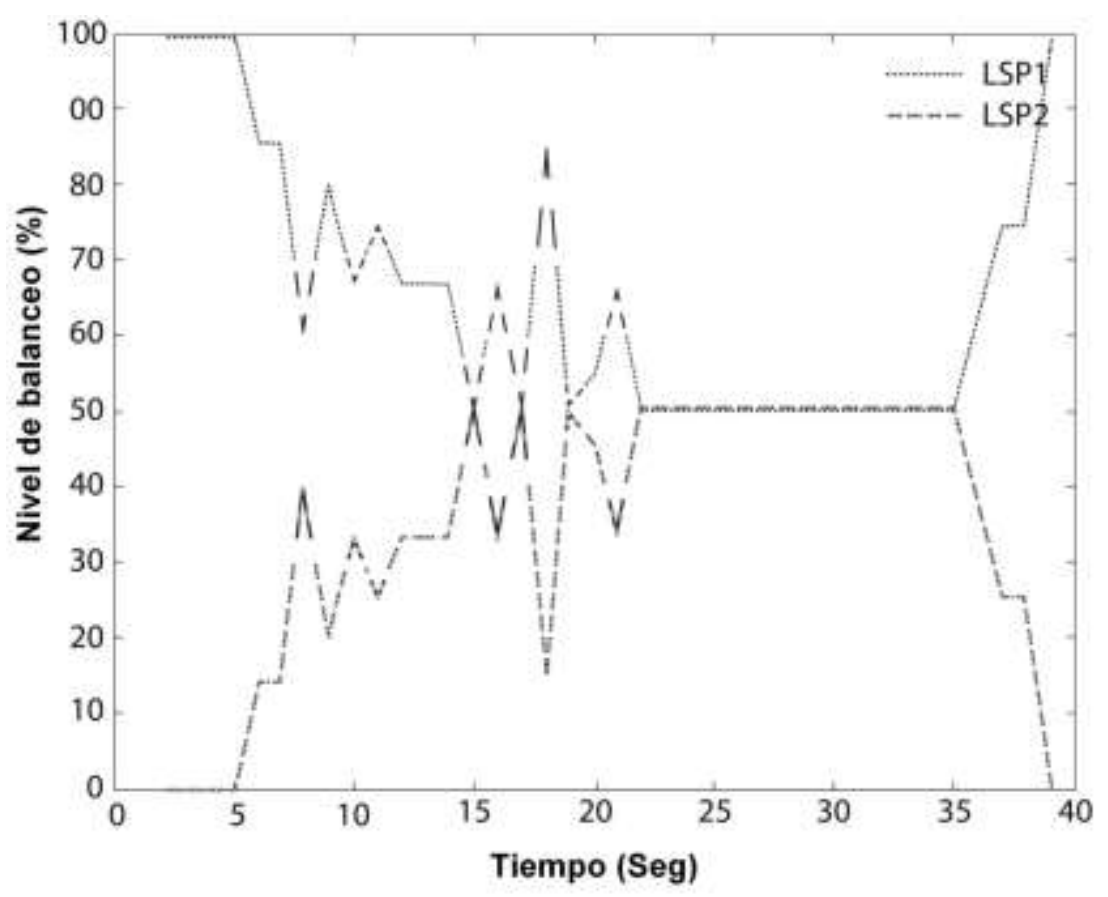

Fig. 12: Inestabilidad en nivel de balanceo $(\alpha=1)$. 
El segundo caso, cuando $\alpha=0.5$ (Fig. 13) la respuesta del algoritmo a los cambios variantes del tráfico en cuanto a ancho de banda solicitadas por el protocolo OSPF-TE (Katz et al., 2003) muestran que aunque existen variaciones en los LSPs no son tan cambiantes, respondiendo con menos oscilaciones si se compara con los de la Fig. 11, debido a que LCM toma en cuenta para el reparto de la carga los valores de carga de flujo calculados con anterioridad.

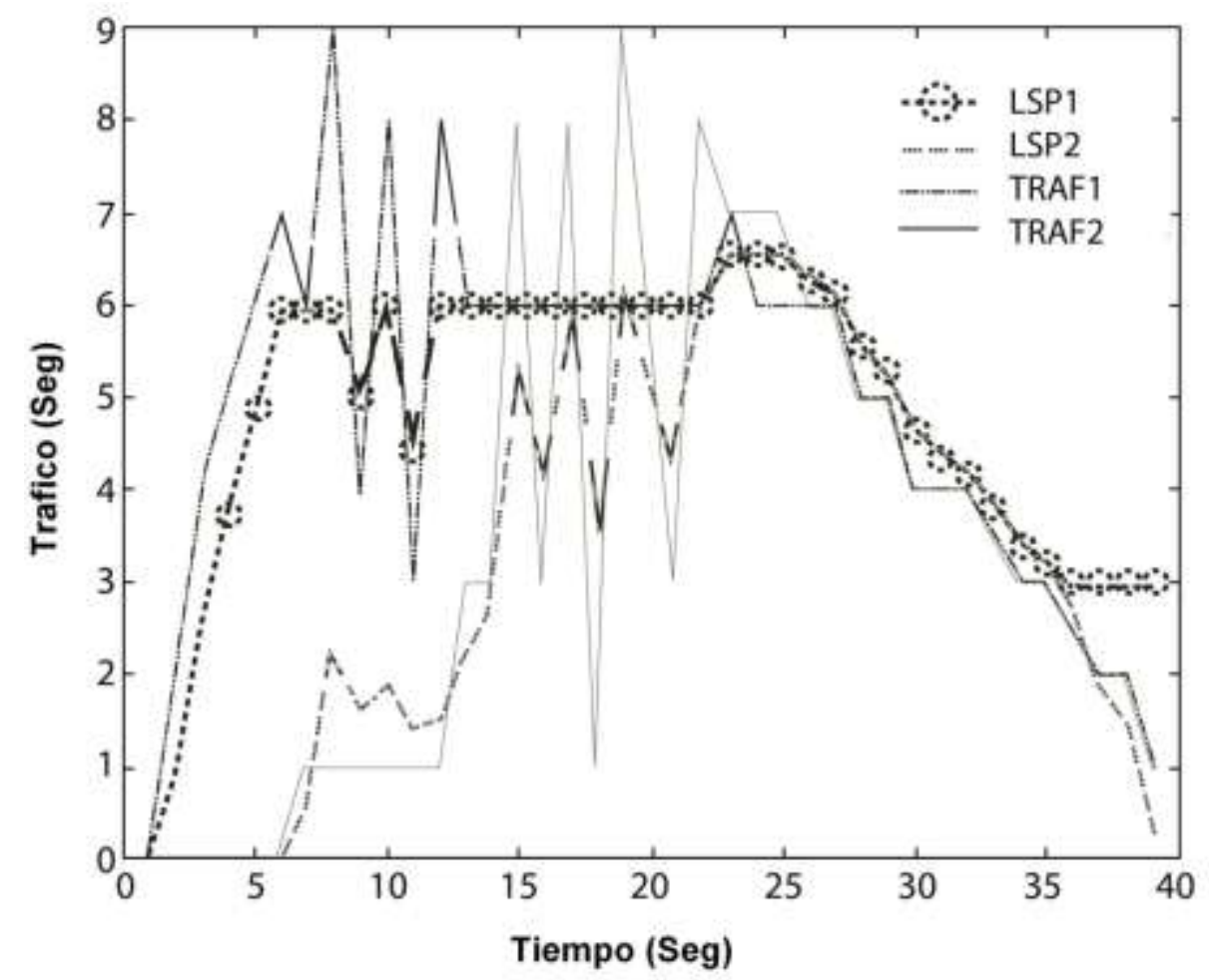

Fig. 13: Inestabilidad en el tráfico por los $\operatorname{LSP}(\alpha=0.5)$.

En la Fig. 14, se refleja la inestabilidad en el porcentaje de balance en menor medida donde la amplitud de las oscilaciones ha disminuido mejorando la estabilidad, pues LCM en este caso toma en cuenta la mitad de las medidas anteriores y la mitad de las actuales en el cálculo final volviendo más uniforme el nivel de balanceo.

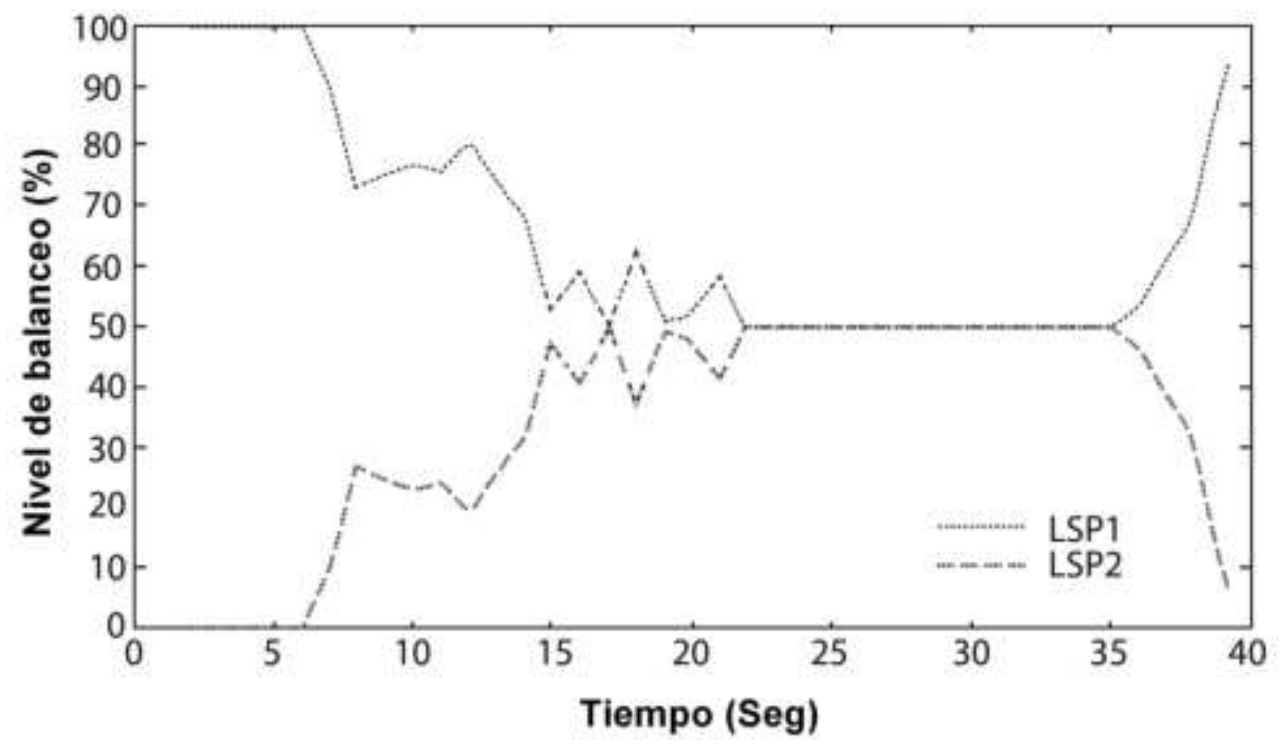

Fig. 14: Inestabilidad en el nivel de balanceo $(\alpha=0.5)$. 
Si $\alpha=0.1$ (Fig. 15) el desempeño en el tiempo es aún más suave, ya que se toman en cuenta en una gran proporción las actualizaciones de tráfico anterior, eliminando totalmente las oscilaciones y produciendo una respuesta estable pero a su vez más lenta e imprecisa frente a las actualizaciones relacionadas con la información del estado de la red en tiempo real. En la Fig. 16, se ve claramente que la respuesta no tiene oscilaciones, ni cambios abruptos mejorando notablemente la estabilidad en comparación con los casos anteriores. La estabilidad mejora claramente, pero se corrobora que es más lenta la respuesta a este tipo de cambios. Para un $\alpha=0.1$ el diagrama de flujo de LCM toma en una proporción del $10 \%$ para las medidas de tráfico actuales y $90 \%$ para las actualizaciones anteriores; como es evidente las medidas actuales de tráfico afectan muy poco la respuesta, dependiendo en gran medida de las anteriores.

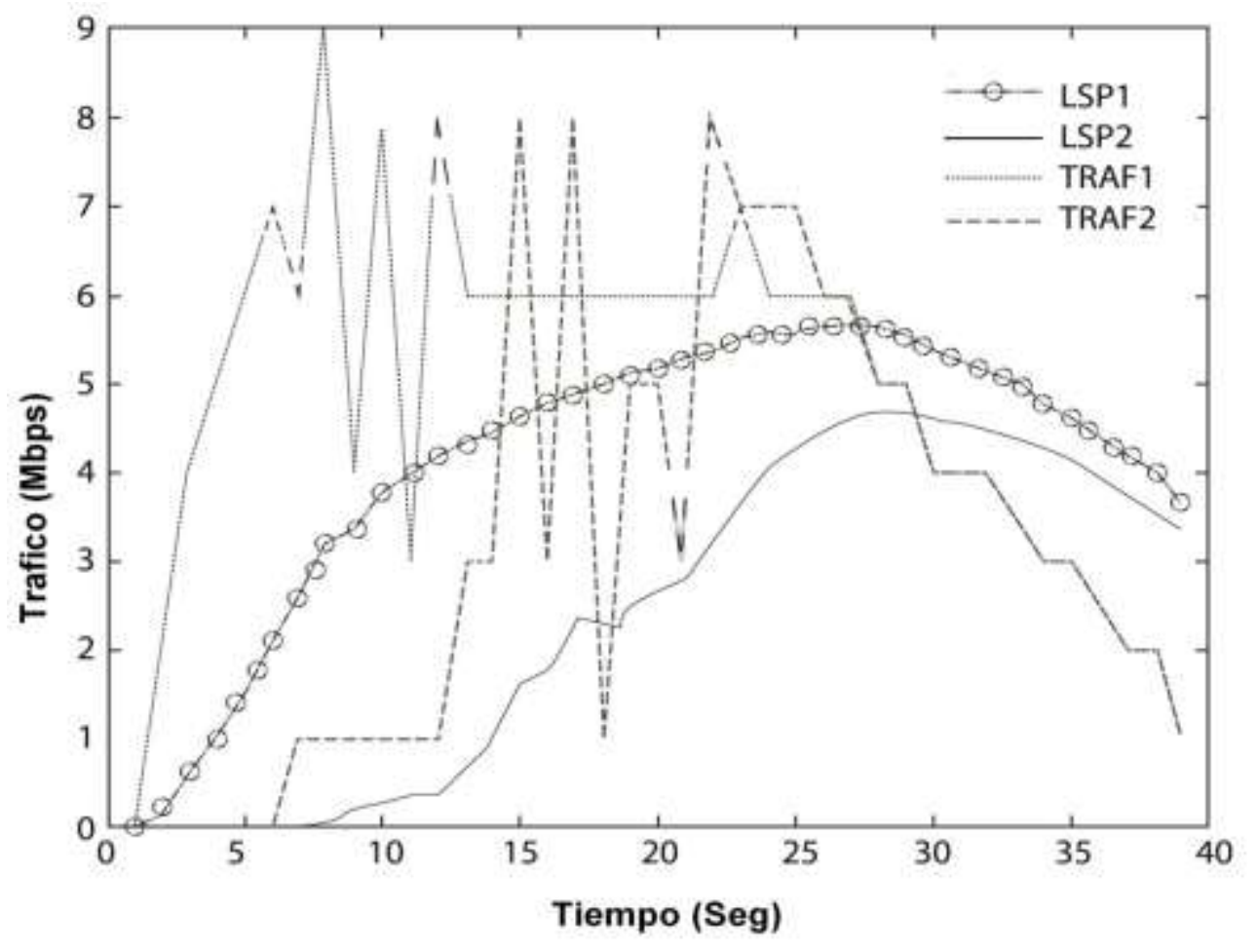

Fig. 15: Inestabilidad en el tráfico por los LSP $(\alpha=0.1)$.

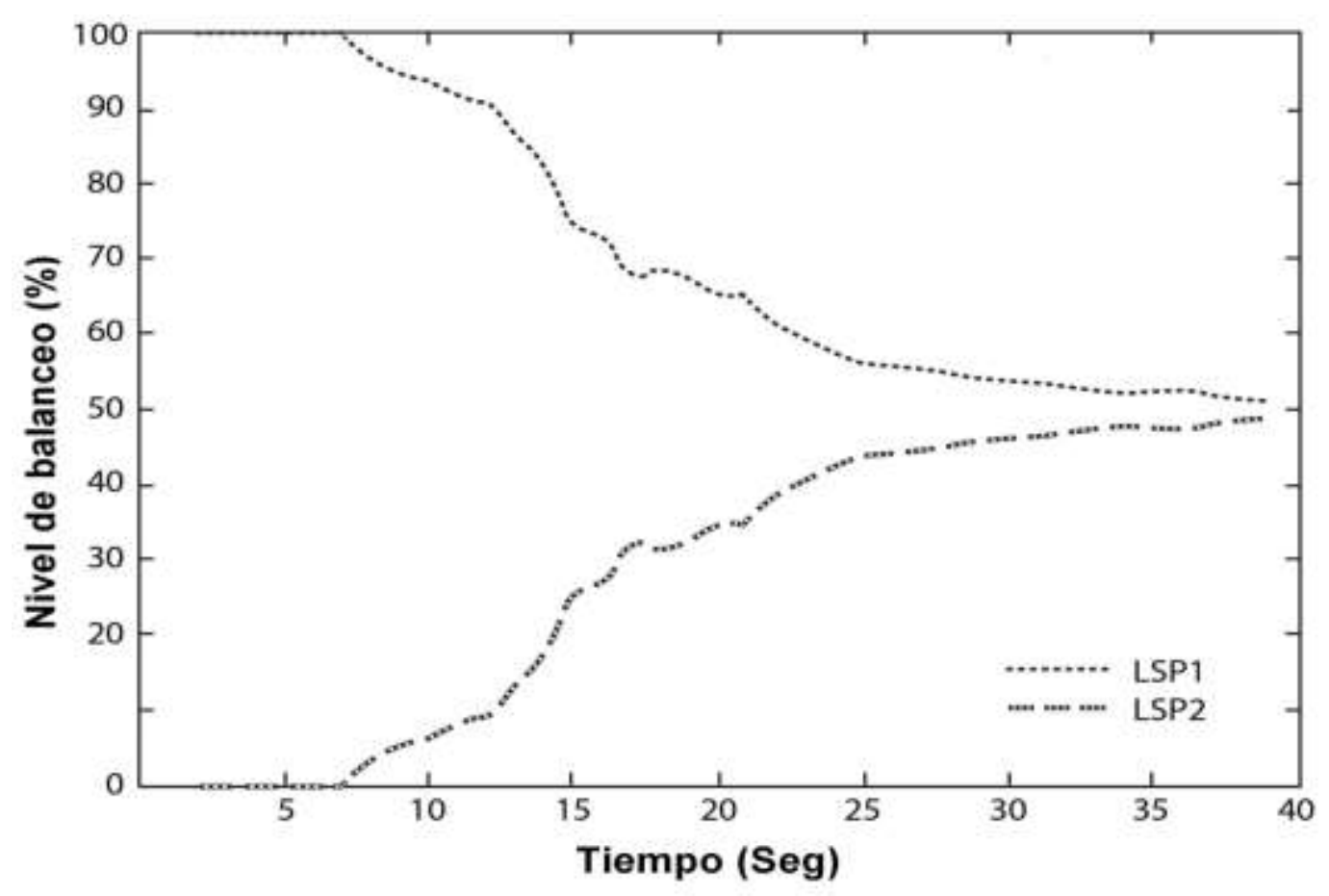

Fig. 16: Estabilidad en el nivel de balanceo $(\alpha=0.1)$. 


\section{CONCLUSIONES}

El balanceo de carga, es una métrica fundamental que debe ser evaluada en la inclusión de nuevas tecnologías de red, pues de ella depende que se haga un uso eficiente de los recursos sin llegar a saturar los enlaces primarios. La utilización de LCM en redes de conmutación GMPLS en los casos estudiados en el artículo da como resultado que desde el punto de vista real sería una buena opción a implementar pues su performance en cuanto a las variables throughput, jitter, balanceo y estabilidad son adecuadas.

LCM es más rápido en responder cuando ocurre algún cambio en el estado de la red (si se compara con MATE en GMPLS), ya que este algoritmo se está ejecutando constantemente, verificando las medidas de la red, además de que OSPF-TE difunde la información del estado de la red cada vez que se producen, de acuerdo a la configuración existente.

Una gran ventaja de LCM desde el punto de vista de la estabilidad, es que con un $\alpha=0.1$ la estabilidad de la red es óptima ya que no experimenta oscilaciones, y como no es un algoritmo iterativo de gradiente no genera problema de divergencia.

\section{REFERENCIAS}

Antti, M., S. Siikavirta., M. Jukka, Comparison of load-balancing approaches for multipath connectivity, Computer Networks, 56(8) 2179-2195 (2012).

Arco, J., A. García., J. A. Carral., G. Ibáñez, BSO algoritmo de reparto de tráfico para MPLS-TE, Universidad de Alcalá, Departamento de Ingeniería Computacional, España (2004).

Arco, J., A. García., E. Castro., J. A. Carral., G. Ibáñez, LCM: A load balance algorithm in MPLS-TE, Universidad de Alcalá, Departamento de Ingeniería Computacional, 2004. [En línea], consultado en Abril 17 de 2012: https://portal.uah.es/portal/page/portal/epd2_profesores/prof28259/publicaciones/gerona06.pdf

Cao, Z., W. Zheng., E. Zegura, Hashing-Based traffic splitting algorithms for internet load balancing, Georgia Institute of Technology, Atlanta (2001).

Chih-Heng, K, How to measure packet loss rate, jitter, and end-to-end delay for UDP-based applications? EE Department, NCKU, 2011. [En línea], consultado en Abril 10 de 2012, disponible en: http://140.116.72.80/ smallko/ns2/tool_en.htm.

Demichelis, C., P. Chimento, IP packet delay variation metric for IP performance metrics (IPPM), RFC 3393, (2002).

El-Alfy, E., Mujahid, S., Selim, S, A Pareto-based hybrid multiobjective evolutionary approach for constrained multipath traffic engineering optimization in MPLS/GMPLS networks, Journal of Network and Computer Applications, 36 (4) 1196-1207 (2013).

Katz, D., K. Kompella., D. Yeung, Traffic engineering (TE) extensions to OSPF, Version 2, RFC 3630 , (2003).

López, D., Hernández, C., Salcedo, O, Redes Multidifusión utilizando Conmutación de Etiquetas multiprotocolo y estándares de Señalización. Revista Información Tecnológica, 23(6) 43-50 (2012).

López, D., N. García., P. Figueroa, Estimación y optimización del balanceo de carga ofrecido por MATE en redes GMPLS, Revista Información tecnológica, 25(2) 47-56 (2014).

Muñoz, W., M. Trujillo, Mecanismos de balanceo de carga en MPLS con RSVP-TE y OSPF, Presentación tesis de grado, Universidad del Cauca (Colombia). [En línea], consultado en Junio 22 de 2012, disponible en: http://artemisa.unicauca.edu.co/ mtrujillo/tesis.swf.

Wenda, N., H. Changcheng, W. Jing., S. Michel, Availability of survivable valiant load balancing (VLB) networks over optical networks, Optical Switching and Networking, 10(3) 274-289 (2013).

Weiqiang, S., X. Zijie., K. Kai., J. Yaohui., G. Wei., H. Weisheng, Performance of label switched path dynamic provisioning in GMPLS Networks, IEEE Communications Magazine, 50(1) 100-105 (2012).

Zhanqi, X., H. Jiangjiang., Z. Zhiqiang., D. Zhe., M. Tao., W. Junping, A novel grooming algorithm with the adaptive weight and load balancing for dynamic holding-time-aware traffic in optical networks, Optical Fiber Technology, 19(5) 392-399 (2013). 